\title{
Inflorescence Identity Gene Alleles Are Poor Predictors of Inflorescence Type in Broccoli and Cauliflower
}

\author{
Joanne A. Labate, Larry D. Robertson, and Angela M. Baldo \\ United States Department of Agriculture, Agricultural Research Service, Plant Genetic Resources Unit, \\ New York State Agricultural Experiment Station, Geneva, NY 14456-0462 \\ Thomas Björkman' ${ }^{1}$ \\ Department of Horticultural Sciences, New York State Agricultural Experiment Station, Cornell \\ University, Geneva, NY 14456-0462
}

\begin{abstract}
ADDITIONAL INDEX WORDS. Brassica oleracea var. botrytis, B. oleracea var. italica, BoAP1-a, BoCAL-a, BoGSL-ELONG, developmental arrest, APETALA-1
\end{abstract}

\begin{abstract}
Broccoli (Brassica oleracea L. var. italica Plenck) and cauliflower (B. oleracea var. botrytis DC) are closely related botanical varieties. The underlying genetic bases of their phenotypic differences from each other are not well understood. A molecular genetic marker enabling $B$. olerace $a$ germplasm curators and breeders to predict phenotype from seeds or seedlings would be a valuable tool. Mutant alleles at flower developmental pathway loci $B o A P 1-a, B o$ $C A L-a$, and glucosinolate biosynthetic pathway locus BoGSL-ELONG have been reported to be associated with a cauliflower phenotype. We surveyed mutant alleles at these three loci in a genetically diverse sample of broccoli and cauliflower accessions from the U.S. Department of Agriculture, Agricultural Research Service (USDA-ARS) Plant Genetic Resources Unit (PGRU) and the University of Warwick, Genetic Resources Unit of Warwick HRI (HRI). Phenotypic and genotypic data were collected for multiple plants per accession during two field seasons. Simple genetic models assuming dominance or codominance of alleles were analyzed. Goodness-of-fit tests rejected the null model that the mutant genotype was associated with a cauliflower phenotype. A correlation analysis showed that BoAP1-a and $B o C A L-a$ alleles or loci were significantly correlated with phenotype but the fraction of variation explained was low, $4.4 \%$ to $6.3 \%$. Adding $B o G S L-E L O N G$ to the analysis improved predictive power using the linear regression procedure, Maximum $R$-square Improvement $\left(\max R^{2}\right)$. In the best three-variable model, only $24.8 \%$ of observed phenotypic variation was explained. Because tested genetic models did not hold robustly for the surveyed accessions, it is likely that there are multiple genetic mechanisms that influence whether the phenotype is broccoli or cauliflower. Our results in commercial cultivars indicate that other genetic mechanisms are more important in determining the horticultural type than are BoAPI-a and BoCAL-a.
\end{abstract}

The cole crops (Brassica oleracea) are characterized by diverse morphological forms that likely resulted from selection for various edible parts. Examples include, leaves that form a head [cabbage (B. oleracea var. capitata L.)], non-heading leafy types \{kale and collard greens (B. oleracea var. acephala DC), chinese kale [B. oleracea var. alboglabra (L.H. Bailey) Musil]\}, an enlarged stem [kohlrabi (B. oleracea var. gongylodes L.)], axillary buds \{brussels sprouts [B. oleracea var. gemmifera (DC) Schultz]\}, and immature inflorescences [broccoli (B. oleracea var. italica), cauliflower (B. oleracea var. botrytis)]. Broccoli and cauliflower are similar in that precociously large inflorescences (flower buds, pedicels, and peduncles in the case of broccoli) constitute the edible part of the plant. This has generated much confusion regarding their distinction in both scientific and popular literature (Kalia and Sharma, 2004). A robust definition was proposed by Gray (1982) based on the relative ontogeny of broccoli vs. cauliflower at marketable maturity. Broccoli heads are a mass of fully differentiated flower buds, while cauliflower crowns (known as curds) consist of proliferated floral meristems, about $90 \%$ of which abort prior to developing into buds (Gray, 1982).

Received for publication 16 Mar. 2006. Accepted for publication 21 June 2006. We thank Susan Sheffer and Paul Kisly for their excellent technical assistance, Drs. Heidi Schwaninger and Lori Hinze for helpful reviews of the manuscript, and Dr. David Astley for seed from the collection at Warwick HRI.

1To whom reprint requests should be addressed; e-mail: tnb1@ cornell.edu
Arguing that the curding character of cauliflower is under major gene control, two lines of evidence were presented by Crisp (1982). First, reproductive fitness of cauliflower is low compared to broccoli. The majority of floral meristems abort, and this excess undifferentiated tissue is particularly susceptible to attack by pathogens. Crisp asserted that it is difficult to imagine the curding trait evolving gradually under these conditions, but that it seemed more plausible that humans discovered a grossly mutated form that was then favored for seed production. Second, Crisp et al. (1975) discovered a single, dominant gene mutation in cauliflower which gave rise to very small curds on vegetatively normal plants (i.e., a reproductive fitness similar to broccoli). This mutation was thought to possibly represent a back mutation to an ancestral allele. In addition, although Crisp's studies of crosses between broccoli and cauliflower did not show evidence of a major gene effect in $F_{1}$ or $F_{2}$, selfs of $F_{8}$ and $F_{9}$ plants showed genetic evidence, albeit recessive, of a single locus difference responsible for curding (Crisp, 1982).

The cauliflower mutant phenotype in the genetic model Arabidopsis thaliana (L.) Heyn is reminiscent of the curding trait in $B$. oleracea var. botrytis, and results from combining recessive mutations in CAULIFLOWER (CAL) and APETELAI (API) genes in the model species (Kempin et al., 1995). A nonsense mutation in the B. oleracea var. botrytis $C A L$ homolog provided additional evidence that $C A L$ and $A P 1$ homologs BoCAL- $a$ and BoAPl- $a$ 
are good candidate genes for influencing expression of curding in cauliflower vs. broccoli (Kempin et al., 1995).

Subsequent molecular and genetic findings supported this model (Smith and King, 2000). Perfect correlations were observed between BoCAL- $a$ and BoAPl-a mutant alleles and curding in a doubled-haploid segregating population that originated by crossing an inbred calabrese broccoli to an $\mathrm{F}_{1}$ cauliflower (i.e., double-mutant plants were full-curding, double-wild-type plants were calabrese broccoli) (Smith and King, 2000).

However, other data support a role for these genes in heading, but not for distinguishing between broccoli and cauliflower. A survey of the BoCAL- $a$ gene in 37 worldwide $B$. oleracea accessions found that the nonsense mutant allele was not strictly associated with cauliflower, or even heading types (Purugganan et al., 2000). Although it was fixed in cauliflower, it was also observed at high frequency in broccoli, and at lower frequencies in the non-heading types kale and wild field cabbage (B. oleracea var. oleracea L.). Patterns of nucleotide variation at the locus implied that the nonsense mutation originated relatively recently and was positively selected by humans in both broccoli and cauliflower. The authors proposed that BoCAL-a mutations have played a role in evolution of altered inflorescence in B. oleracea in general. Another survey of a diverse set of 206 crop B. oleracea accessions showed the BoCAL- $a$ mutant allele to be at very high frequency in curding types, although 17 curding accessions were heterozygous and two lacked the mutant allele (Smith and King, 2000). If there is only a general correlation, these genes will have no diagnostic value, and the proposed function of the genes will need to be revisited.

A major aliphatic glucosinolate gene, BoGSL-ELONG, was isolated from $B$. oleracea based on similarity to its $A$. thaliana homolog GS-ELONG (Li and Quiros, 2002). The gene product is necessary for the addition of four-carbon side chains to glucosinolates (4C GSL). These side chains occur in broccoli but not in cauliflower. A nonfunctional allele of BoGSL-ELONG from white cauliflower was associated with a 30-bp deletion, which allowed Li and Quiros (2002) to develop a codominant molecular marker for 4C GSL.

A molecular genetic marker that would enable $B$. oleracea germplasm curators and breeders to predict phenotype without growing a plant to maturity would be valuable. Expression of a heading phenotype can take upwards of 2 years or more under field conditions. Accessions held by germplasm repositories are sometimes insufficiently documented. For example, within-accession phenotypic variation can encompass broccoli, cauliflower and intermediate types while associated passport data only indicates the most prevalent type (unpublished observations). In cauliflower breeding, transferring a desirable trait directly from broccoli can impose great difficulty in later recovering the cauliflower phenotype.

Our objective was to examine applicability of the model that mutations in the $B o C A L-a$ and $B o A P 1-a$ loci confer the cauliflower inflorescence type on cole crops with heading inflorescences. First, we tested whether the model applies to currently cultivated broccoli and cauliflower. Second, we tested whether these loci, together with a cauliflower-specific allele at BoGSL-ELONG, can be used to classify $B$. oleracea germplasm as broccoli or cauliflower for curatorial or breeding purposes. This is the first study to test correlations between genotype and curding within open-pollinated populations phenotypically segregating for broccoli and cauliflower.

\section{Materials and Methods}

Plant Growth. Seed of accessions were started in plug flats, grown in the greenhouse for 4 weeks, then transplanted in mid-May into plastic-mulch beds in the field at the Wellington Research Farm in Geneva, N.Y. Accessions were grown in rows spaced $0.9 \mathrm{~m}$ between rows and $0.6 \mathrm{~m}$ between plants. In both 2001 and 2002, 20N-4.4P-8.3K fertilizer was applied once at a rate of $3.36 \mathrm{~kg} \cdot \mathrm{ha}^{-1}$. Each year, glyphosate (Roundup; Monsanto, St. Louis) was applied once at the rate of $2.37 \mathrm{~L} \cdot \mathrm{ha}^{-1}$ between rows to control weeds. To control diseases, chlorothalonil (Bravo; Syngenta Crop Protection, Greensboro, N.C.) was applied once at $1.12 \mathrm{~kg} \cdot \mathrm{ha}^{-1}$ (a.i.). Insects were controlled through two applications of carbaryl (Sevin XLR; Bayer CropProtection, Research Triangle Park, N.C.) at the rate of $2.37 \mathrm{~L} \cdot \mathrm{ha}^{-1}$ (a.i.) and of endosulfan (Endosulfan 3 EC; Helena Chemical Co., Collierville, Tenn.) at the rate of $2.37 \mathrm{~L} \cdot \mathrm{ha}^{-1}$ (a.i.).

Plant materials. In order to study among-accession variation, during field season 1 (2001) 30 plants per accession were planted for each of $40 \mathrm{~B}$. olerace a accessions. These consisted of 27 PGRU accessions identified by passport data as broccoli or kale, four PGRU accessions identified as cauliflower, and nine HRI accessions indicating "broccoli" in their common name (Table 1). Phenotypic observations were collected for all plants and from three (for $F_{1}$ populations) to 30 plants were genotyped per accession. To study within-accession variation, during the second field season (2002) 48 plants per accession were planted for each of 10 accessions that had segregated for genotype and/or phenotype during 2001. Phenotypic observations were collected for all plants and 0 to 40 plants per accession were genotyped in field season two.

Phenotypic Characterization. Plants were inspected twice weekly and assessed at the stage most closely corresponding to harvest maturity. Generally, this stage is just before the head begins to separate as part of the onset of bolting. The stage of developmental arrest was scored for each plant on a seven-point scale with a photographic reference card and based on measurements of sepal length in $\mathrm{mm}$. The arrest stages varied from pure reproductive meristem (cauliflower) through floral primordia, immature buds, mature buds (broccoli) and absence of arrest (nonheading). Arrest stages were coded into one of three phenotypic classes (arrest 1 and $2=$ phenotype $1=$ broccoli; arrest 3 and 4 $=$ phenotype $2=$ intermediate; arrest 5,6 , and $7=$ phenotype 3 = cauliflower) as shown in Fig. 1 .

DNA ANALYSIS. Samples of 0.050 to $0.100 \mathrm{~g}$ of succulent young leaf tissue were taken from each plant and genomic DNA was isolated using a modified CTAB method (Colosi and Schaal, 1993). All PCR assays included positive (known genotypes) and negative (noDNA) controls. For genotyping the BoAPl-a locus, a 5 'length polymorphism was used to distinguish mutant from wildtype alleles (Smith and King, 2000). An approximately 100-bp fragment was amplified with primers AP1 PF and AP15PR (Smith and King, 2000), the forward primer was fluorescently labeled with IR800 dye. The amplicons were separated by polyacrylamide gel electrophoresis (GeneScan; LI-COR, Lincoln, Nebr.) using known wild-type and mutant genotypes as controls to detect an approximately four base-pair deletion in the cauliflower mutant allele. BoCAL- $a$ was amplified from genomic DNA using CAL4F and CAL16R primers according to published protocols (Smith and King, 2000). Because PCR yields were sometimes low, a 50- $\mu \mathrm{L}$ second round of amplification of a 750-bp fragment was performed 
Table 1. Passport phenotype and observed heading arrest stage in 31 Brassica oleracea accessions from the USDA-ARS Plant Genetic Resources Unit and nine B. oleracea accessions from the Warwick HRI Genetic Resources Unit when grown in the field in Geneva, N.Y. during the summer, and available allele frequencies at BoCAL-a, BoAPl-a, and BoGSL-ELONG.

\begin{tabular}{|c|c|c|c|c|c|c|c|c|c|c|c|c|c|c|c|c|c|c|c|c|c|c|c|}
\hline \multirow[b]{4}{*}{ Accession } & \multirow{4}{*}{$\begin{array}{l}\text { Passport } \\
\text { Phenotype }\end{array}$} & \multicolumn{11}{|c|}{2001} & \multicolumn{11}{|c|}{2002} \\
\hline & & \multirow{3}{*}{ Arrest ${ }^{x}$} & \multicolumn{10}{|c|}{ Genotype $^{z}$} & \multirow[b]{3}{*}{ Arrest $^{\mathrm{x}}$} & \multicolumn{10}{|c|}{ Genotype $^{z}$} \\
\hline & & & \multirow[b]{2}{*}{$n$} & \multicolumn{3}{|c|}{$B o C A L-a$} & \multicolumn{3}{|c|}{$B o A P 1-a$} & \multicolumn{3}{|c|}{ BoGSL-ELONG } & & \multirow[b]{2}{*}{$n$} & \multicolumn{3}{|c|}{$B o C A L-a$} & \multicolumn{3}{|c|}{$B o A P 1-a$} & $B o G S$ & $S L-E L C$ & \\
\hline & & & & $\% \mathrm{~W}$ & $\% \mathrm{M}$ & $n$ & $\% \mathrm{~W}$ & $\% \mathrm{M}$ & $n$ & $\% \mathrm{~W}$ & $\% \mathrm{M}$ & $n$ & & & $\% \mathrm{~W}$ & $\% \mathrm{M}$ & $n$ & $\% \mathrm{~W}$ & $\% \mathrm{M}$ & $n$ & $\% \mathrm{~W}$ & $\% \mathrm{M}$ & $n$ \\
\hline G 28828 & cauliflower & $\mathrm{na}^{\mathrm{w}}$ & 10 & 100 & 0 & 10 & & & & & & & & & & & & & & & & & \\
\hline G 30009 & broccoli & $2.4^{\mathrm{v}}$ & 22 & 100 & 0 & 28 & 0 & 100 & 22 & 2 & 98 & 22 & & & & & & & & & & & \\
\hline G 30413 & broccoliu $^{u}$ & 2.0 & 8 & 100 & 0 & 8 & 17 & 83 & 3 & 25 & 75 & 2 & & & & & & & & & & & \\
\hline G 30414 & broccoli $^{\mathrm{u}}$ & 2.0 & 2 & 100 & 0 & 3 & 0 & 100 & 3 & 67 & 33 & 3 & & & & & & & & & & & \\
\hline G 30415 & broccoli $^{\mathrm{u}}$ & 2.0 & 3 & 100 & 0 & 3 & & & & 100 & 0 & 3 & & & & & & & & & & & \\
\hline G 30416 & broccoliu $^{u}$ & 2.0 & 29 & 100 & 0 & 30 & 0 & 100 & 29 & 50 & 50 & 16 & & & & & & & & & & & \\
\hline G 30439 & broccoli $^{\mathrm{u}}$ & 4.0 & 10 & 100 & 0 & 10 & & & & & & & & & & & & & & & & & \\
\hline G 30769 & cauliflower $^{\mathrm{u}}$ & 2.7 & 3 & 0 & 100 & 3 & 0 & 100 & 3 & & & & 4.4 & 37 & 0 & 100 & 9 & 0 & 100 & 9 & 44 & 56 & 8 \\
\hline G 30771 & broccoli $^{u}$ & 2.0 & 1 & 100 & 0 & 3 & & & & & & & & & & & & & & & & & \\
\hline G 30774 & broccoli $^{u}$ & 2.0 & 3 & 100 & 0 & 3 & & & & 50 & 50 & 1 & & & & & & & & & & & \\
\hline G 30778 & broccoli $^{u}$ & 2.0 & 3 & 0 & 100 & 3 & 0 & 100 & 3 & 83 & 17 & 3 & & & & & & & & & & & \\
\hline G 30928 & broccoli & $4.0^{t}$ & 7 & 80 & 20 & 10 & 0 & 100 & 2 & 0 & 100 & 2 & 3.0 & 38 & 56 & 44 & 40 & 17 & 83 & 38 & 3 & 97 & 39 \\
\hline G 31824 & broccoli & $2.0^{\mathrm{v}}$ & 27 & 20 & 80 & 30 & 0 & 100 & 28 & & & & 3.3 & 45 & 0 & 100 & 7 & 0 & 100 & 39 & 47 & 53 & 38 \\
\hline G 31825 & broccoli & $2.1^{\mathrm{v}}$ & 8 & 100 & 0 & 10 & & & & & & & & & & & & & & & & & \\
\hline G 32210 & broccoli & $4.0^{\mathrm{t}}$ & 3 & 0 & 100 & 3 & 0 & 100 & 3 & 100 & 0 & 3 & 2.2 & 47 & 0 & 100 & 5 & 0 & 100 & 8 & 100 & 0 & 7 \\
\hline G 32213 & broccoli & 2.0 & 10 & 100 & 0 & 10 & 5 & 95 & 10 & 100 & 0 & 10 & & & & & & & & & & & \\
\hline PI 115881 & cauliflower & 1.6 & 27 & 33 & 66 & 27 & 54 & 46 & 24 & 2 & 98 & 23 & 2.1 & 40 & 27 & 73 & 39 & 50 & 50 & 32 & 0 & 100 & 31 \\
\hline PI 188561 & Chinese kale & $2.0^{\mathrm{s}}$ & 3 & 100 & 0 & 8 & & & & & & & & & & & & & & & & & \\
\hline PI 231210 & broccoli & $4.6^{\mathrm{r}}$ & 8 & 20 & 80 & 10 & 0 & 100 & 5 & 17 & 83 & 6 & & & & & & & & & & & \\
\hline PI 234599 & cauliflower & $\mathrm{na}^{\mathrm{q}}$ & 5 & 0 & 100 & 10 & & & & & & & & & & & & & & & & & \\
\hline PI 249556 & Chinese kale & 1.0 & 22 & 100 & 0 & 29 & 0 & 100 & 21 & 93 & 7 & 22 & & & & & & & & & & & \\
\hline PI 285596 & broccoli & $6.0^{\mathrm{p}}$ & 8 & 0 & 100 & 9 & & & & & & & & & & & & & & & & & \\
\hline PI 291997 & broccoli & $\mathrm{na}^{\mathrm{w}}$ & 9 & 0 & 100 & 10 & & & & & & & & & & & & & & & & & \\
\hline PI 430580 & broccoli & $5.8^{\mathrm{r}}$ & 8 & 0 & 100 & 10 & 0 & 100 & 8 & 0 & 100 & 8 & & & & & & & & & & & \\
\hline PI 441510 & broccoli & $2.4^{\mathrm{v}}$ & 8 & 75 & 25 & 10 & 0 & 100 & 9 & 25 & 75 & 10 & 2.6 & 41 & 59 & 41 & 35 & 0 & 100 & 34 & 11 & 89 & 32 \\
\hline PI 443022 & broccoli & $5.8^{\mathrm{p}}$ & 10 & 100 & 0 & 10 & 0 & 100 & 10 & 39 & 61 & 9 & & & & & & & & & & & \\
\hline PI 462206 & broccoli & 2.0 & 10 & 100 & 0 & 10 & 60 & 40 & 10 & 30 & 70 & 10 & & & & & & & & & & & \\
\hline PI 462208 & broccoli & $2.0^{\circ}$ & 10 & 100 & 0 & 10 & & & & & & & & & & & & & & & & & \\
\hline PI 462209 & broccoli & $3.0^{\mathrm{n}}$ & 8 & 90 & 10 & 10 & & & & & & & 1.5 & 22 & & & & & & & & & \\
\hline PI 462210 & broccoli & 1.0 & 1 & 75 & 25 & 10 & & & & & & & $\mathrm{na}^{\mathrm{w}}$ & 4 & & & & & & & & & \\
\hline PI 462222 & broccoli & $4.0^{t}$ & 6 & 28 & 72 & 9 & & & & & & & 3.1 & 17 & & & & 0 & 100 & 12 & 0 & 100 & 10 \\
\hline HRI 5295 & $\begin{array}{l}\text { purple head } \\
\text { broccoli }\end{array}$ & $4.0^{t}$ & 21 & 48 & 52 & 26 & 0 & 100 & 21 & & & & $4.0^{t}$ & 39 & 33 & 67 & 6 & 0 & 100 & 39 & 13 & 87 & 39 \\
\hline HRI 4707 & Calabrese & $2.5^{\mathrm{s}}$ & 6 & 100 & 0 & 7 & & & & & & & & & & & & & & & & & \\
\hline HRI 3553 & $\begin{array}{l}\text { sprouting } \\
\text { broccoli }\end{array}$ & $\mathrm{na}^{\mathrm{w}}$ & 9 & 28 & 72 & 9 & & & & & & & & & & & & & & & & & \\
\hline HRI 3575 & broccoli & $2.0^{\mathrm{m}}$ & 8 & 19 & 81 & 8 & & & & & & & & & & & & & & & & & \\
\hline HRI 7519 & broccoli & 2.0 & 3 & 100 & 0 & 3 & & & & & & & & & & & & & & & & & \\
\hline HRI 8196 & broccoli & $\mathrm{na}^{\mathrm{w}}$ & 6 & 25 & 75 & 6 & & & & & & & & & & & & & & & & & \\
\hline HRI 3569 & broccoli & $\mathrm{na}^{\mathrm{w}}$ & 3 & 0 & 100 & 3 & & & & & & & & & & & & & & & & & \\
\hline HRI 8680 & broccoli & $\mathrm{na}^{\mathrm{w}}$ & 5 & 100 & 0 & 5 & & & & & & & & & & & & & & & & & \\
\hline HRI 10674 & broccoli & $\mathrm{na}^{\mathrm{w}}$ & 6 & 58 & 42 & 6 & & & & & & & & & & & & & & & & & \\
\hline
\end{tabular}

${ }^{2}$ Frequencies of $\mathrm{M}=$ mutant allele, and $\mathrm{W}=$ wild-type allele.

${ }^{\mathrm{y} B}$ Broccoli, purple head broccoli and calabrese are B. oleracea var. italica; cauliflower is B. oleracea var. botrytis; chinese kale is B. oleracea var. alboglabra.

${ }^{x}$ Mean phenotype in the field. 1 = wild cabbage, unbranched inflorescence with large flower buds; $2=$ broccoli, uniformly medium-sized flower buds; $3=$ rough broccoli, numerous undersized flower buds; $4=$ purple cauliflower, small flower buds; $5=$ ricy cauiflower, floral primordia; $6=$ mildly ricy cauliflower; some floral primordia; 7 = cauliflower, inflorescence meristem (see Fig. 1).

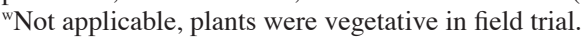

${ }^{v}$ Mixed broccoli and cauliflower in field trial.

${ }^{\text {u }} \mathrm{F}_{1}$ hybrid accession, phenotype is expected to be uniform.

'Purple cauliflower (intermediate) in field trial.

${ }^{\mathrm{s}}$ Brussels sprouts and broccoli in field trial.

${ }^{\mathrm{r}}$ Cauliflower and one purple cauliflower in field trial.

${ }^{q}$ Waxy green and vegetative in field trial.

${ }^{\mathrm{P}}$ Cauliflower in field trial.

${ }^{\circ}$ Two broccoli and eight vegetative in field trial.

"Broccoli, cauliflower, and kale in field trial.

${ }^{\mathrm{m}}$ One broccoli and seven vegetative in field trial.

similar to the first round except that $1-\mu \mathrm{LPCR}$ product and nested primers CALnestF 5'-TCTTATCTTTGAGTTTGTGAAGA-3' and CALnestR 5'-TGTGCACATATGCTCATGATT-3' were substituted. The second round thermoprofile was $94^{\circ} \mathrm{C}, 1 \mathrm{~min}$.; $57{ }^{\circ} \mathrm{C}, 1 \mathrm{~min}$.; $72{ }^{\circ} \mathrm{C}, 1 \mathrm{~min}$.; repeated 35 times. PCR products were cleaned by ethanol precipitation and quantified on a Tecan Spectrafluor (Tecan Group Ltd., Zurich, Switzerland) using a FluoReporter kit (Invitrogen, Carlsbad, Calif.). Then, $500 \mathrm{ng}$ of PCR product was subjected to restriction digest using Spe1
(Promega, Madison, Wis.) and visualized on 2\% agarose gels. The wild-type allele migrates at approximately $750 \mathrm{bp}$, the mutant allele digests and migrates as two fragments at $640 \mathrm{bp}$ and $110 \mathrm{bp}$, and a heterozygote shows all three bands. Alternatively, $50 \mu \mathrm{L}$ of first round PCR product was cleaned using QuickStep 2 cartridges (Edge BioSystems, Gaithersburg, Md.), sequenced in forward and reverse directions using CALnest primers, and electrophoresed on 3100 Genetic Analyzer (Applied Biosystems, Foster City, Calif.). Sequence trace files were visually inspected to 


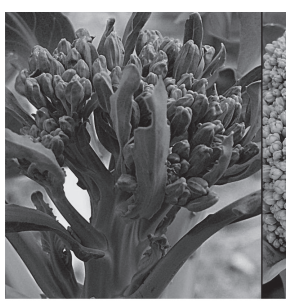

1. Wild cabbage

\begin{abstract}
2. Broccoli
\end{abstract}
3. Rough broccoli

\begin{abstract}
4. Purple cauliflower 5. Ricy cauliflower
Arrest stage score

Phenotype code
\end{abstract}

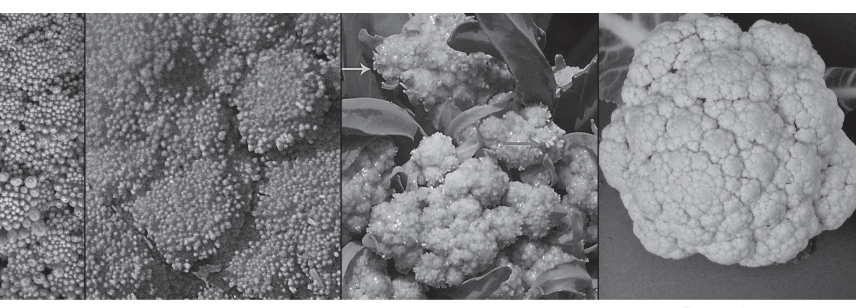

6. Mildly ricy

7. Cauliflower

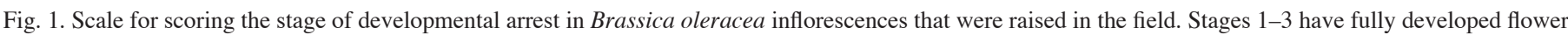

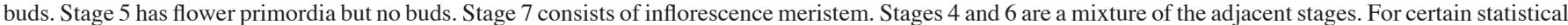
analyses, the phenotypes were consolidated into three classes using the phenotype code on the lower part of the figure.

determine whether the nucleotide site diagnostic of a mutant allele (Kempin et al., 1995) was homozygous T (mutant), homozygous $\mathrm{G}$ (wild type), or heterozygous. BoGSL-ELONG was genotyped on $2 \%$ agarose by PCR amplifying a codominant marker using primers IPM9 and IPM2, which detects a 30-bp deletion in intron 1 in cauliflower ( $\mathrm{Li}$ and Quiros, 2002). To verify allele identity and elucidate the sequence of the indel, five random plants from each of homozygous wild type, heterozygous, and homozygous mutant BoGSL-ELONG genotypes were sequenced on a 3100 Genetic Analyzer in forward and reverse directions using IPM9 and IPM2 primers.

Statistical analysis. All statistical analyses were performed using SAS (version 8; SAS Institute Inc., Cary, N.C.). Year 1 data: A goodness-of-fit ( $G$-test) (Sokal and Rohlf, 1981) was used to test whether the number of mutant alleles $(0,1$, or 2$)$ predicted phenotype $(1,2$, or 3$)$ for $B o C A L-a, B o A P 1-a$, and BoGSL-ELONG separately (single locus, additive allele model). This model assumes that a homozygous mutant genotype is associated with cauliflower, a heterozygote displays an intermediate phenotype, and a homozygous wild-type plant is associated with broccoli. The $G$-test was also used to test, for BoCAL- $a$ and BoAP1- $a$ together (when both were available), whether the number of homozygous mutant loci $(0,1$, or 2$)$ predicted phenotype (additive over loci, dominant-within-locus model). Under this model broccoli would contain no homozygous mutant loci, intermediate phenotypes would contain one homozygous mutant locus, and cauliflower would be homozygous mutant at both loci. The Pearson correlation coefficient $r$ (Sokal and Rohlf, 1981) was used to test whether number of mutant alleles at BoCAL- $a$ and BoAP1- $a$ separately $(0,1$, or 2$)$ or together $(0,1,2,3$, or 4$)$ was correlated with phenotype. This model tested whether number of mutant alleles and degree of curding were positively correlated without specifying which genotypes predict which phenotypes. To examine whether prediction of phenotype was improved by incorporating knowledge of the genotype at a third locus, BoGSL-ELONG, the linear regression procedure max $R^{2}$ (SAS Institute Inc.) was used to model the percent phenotypic variation explained by each of five variable factors: i) $B o C A L-a$ additive alleles; ii) $B o A P 1-a$ additive alleles; iii) $B o G S L-E L O N G$ additive alleles; iv) mutant loci $(0,1,2$, or 3$)$ assuming dominance within a locus and additivity over loci; and v) mutant alleles ( 0 , $1,2,3,4,5$, or 6 ) assuming additivity within and over loci. The max $R^{2}$ procedure identifies the factor that explains the highest proportion of phenotypic variance and reports the results in the form of an analysis of variance (ANOVA) of the best one-variable model. It proceeds to reanalyze and report the best two-variable model, the best three-variable model, etc., for the data until the model cannot be further improved in terms of percent phenotypic variation explained. At each step, $\max R^{2}$ compares all possible combinations of variables and chooses which combination yields the largest $R^{2}$, independently of the previous steps.

Year 2 data: Within-accession genotypic and phenotypic variation was studied for the plants grown in 2002. Because of smaller sample sizes and associated lessening of statistical power relative to the year 1 data, fewer tests were performed. Only three (PI 441510, PI115881, and G 30928) of the 10 accessions provided sufficient data showing segregation for both phenotype and $B o C A L-a$ genotype in year 2. For these three accessions a regression analysis was used to test the association of $B o C A L-a$ and $B o A P 1-a$ loci on phenotype separately or together assuming additivity of mutant alleles within and across loci. The additional seven accessions assayed in 2002 were evaluated qualitatively.

\section{Results}

Plant phenotypes in the diverse array of $B$. olerace a accessions in this study varied from non-heading leafy types, to broccoli, to cauliflower when grown in the field during 2001 and 2002 in Geneva, N.Y. (Table 1). This phenotypic variation was used to test the value of three putative genetic predictors. Mutant and wildtype allele frequencies were estimated for $B o C A L-a, B o A P 1-a$, and BoGSL-ELONG (Table 1).

Among-accession variation. $G$-tests for $B o C A L-a$ and $B o A P 1-a$ separately and $B o C A L-a$ plus $B o A P 1-a$ together were highly statistically significant (Fig. 2). Values of the $G$-statistic greater than the critical value $G_{.01[2]}=9.2$ are rejection of the model that alleles are associated with their respective predicted phenotypes. Thus, the null hypotheses that genotypes at one locus (assuming additivity of alleles) or at both loci taken together (assuming dominance within a locus and additivity over loci) fit predicted phenotypes can be rejected with high confidence. Correlation coefficients between curding phenotype and number of BoCAL- $a$ mutant alleles $(n=291, r=0.235, P<0.0001)$, BoAP1- $a$ mutant alleles $(n=211, r=0.210, P=0.0022)$, mutant $B o C A L-a$ plus BoAPl- $a$ alleles $(n=211, r=0.2485, P=0.0003)$, and mutant BoCAL- $a$ plus BoAP1- $a$ loci $(n=211, r=0.250, P=$ $0.0002)$ were all highly statistically significant but explained a relatively small proportion of the observed phenotypic variation $\left(r^{2}\right.$ $=4.4 \%$ to $6.3 \%)$. BoGSL-ELONG was included in the analyses to see if predictive power could be improved. The best one-variable 


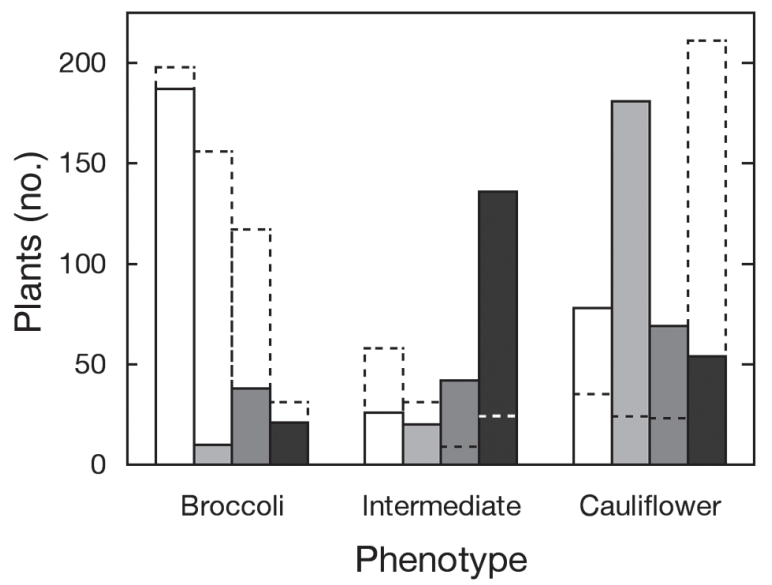

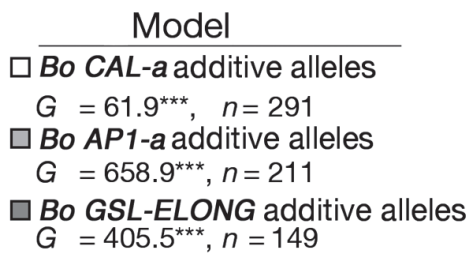

Bo CAL-a plus Bo AP1-a, dominant

within locus, additive over loci

$G=195.5^{* * *}, n=211$

Expected value

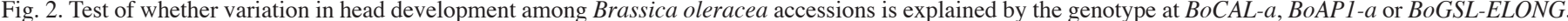

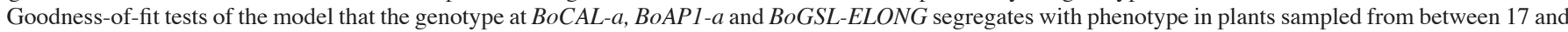
40 B. oleracea accessions from the collections of the USDA-ARS Plant Genetic Resources Unit and the Warwick HRI Genetic Resources Unit. The genotype was

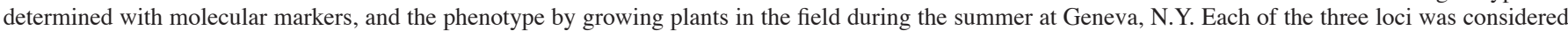
individually as codominant. In addition the model that $B o C A L-a$ and $B o A P 1$ - $a$ have overlapping function was tested by considering each locus dominant but the action of each locus additive. Values of the $G$ statistic greater than the critical value are rejection of the model that recessive mutant alleles segregate with curding.

model when testing the five variable factors $B o C A L-a, B o A P 1-a$, BoGSL-ELONG, number of mutant loci $(0,1,2$, or 3$)$ and number of mutant alleles $(0,1,2,3,4,5$, or 6$)$ selected the variable "number of mutant loci", assuming dominance within a locus and additivity across loci, as indicated by $\max R^{2}=0.1780, \mathrm{df}=$ $141, P<0.0001$. The addition of the variable "BoGSL-ELONG" (assuming additive alleles) gave the best two-variable model $\left(R^{2}=\right.$ $0.2402, \mathrm{df}=141, P<0.0001)$. Further improvement of the model was possible with the addition of "number of mutant alleles" $\left(R^{2}\right.$ $=0.2478$, df $=141, P<0.0001)$. No further improvement over the three-variable model was possible.

WiTHIN-ACCESSION VARIATION. During the 2002 field season, we grew 10 accessions that had segregated either for phenotype, genotype, or both during 2001. Three of the 10 accessions (PI 441510, PI 115881, and G 30928) varied in head type and segregated at $B o C A L-a$ or both $B o C A L-a$ and $B o A P 1-a$ in 2002. A regression analysis of genotype vs. phenotype was performed for $B o C A L-a$ alone, $B o A P 1-a$ alone, and for the two loci combined for these three accessions (Table 2). The regression analysis assumed additivity of the mutant allele within and between loci (i.e., a plant could carry zero to four mutant alleles). In this model, if carrying a mutant allele is associated with a more arrested phenotype then the slope of the regression $(\beta)$ is negative. Therefore, positive values of $\beta$ will never be significant in a one-tailed test. The $B o C A L-a$ geno- type was statistically significant $(P=0.02)$ and explained $16.8 \%$ of the phenotypic variation for PI 441510, 'Ramoso'. A similar result (not shown) was obtained when assuming dominance of the wild-type allele within and across loci $\left(R^{2}=0.189, P=0.01\right)$. The mutant allele for $B o A P 1-a$ was fixed for plants sampled from PI 441510. For PI 115881, an unnamed cauliflower, all $\beta$ values were positive, lending no support for the influence of $B o C A L-a$ and/or BoAP1- $a$ on phenotype in this accession. For G 30928, 'Cavolo broccolo precoce', the effect of the loci was apparently additive and in the correct direction, but nonsignificant and with small $r^{2}$ (Table 2). Four accessions assayed during 2002 gave qualitative results. HRI 5295, a purple cauliflower from southern Italy, had a phenotype intermediate between broccoli and cauliflower. Heads were uniform floral primordia, neither meristem nor floral buds. This cultivar segregated at BoCAL- $a$ (Table 1) but there were no phenotypic differences between wild-type and mutant genotypes. Two accessions, G 31824 (unnamed broccoli from China) and G 30769 'Green Harmony F1' were monomorphic for mutant alleles at both $B o C A L-a$ and $B o A P 1-a$ although they varied in phenotype. $\mathrm{G} 31824$ showed a large proportion of broccoli and purple cauliflower phenotypes. The F1 hybrid cultivar Green Harmony F1 exhibited substantial phenotypic variation in arrest, tending to be cauliflower-like for heads maturing in July and broccoli-like for heads maturing in August (Fig. 3). The

Table 2. Test of whether variation in head development within segregating Brassica oleracea accessions is explained by the genotype at BoCAL- $a$ and BoAP1- $a$. Within-line regression analyses for three accessions that segregated for genotype and also varied for heading phenotype when grown in the field during 2002. A significant effect of the locus would cause a negative slope $(\beta)$ of the regression between the number of wildtype alleles and the phenotypic score. For each accession, the first line represents the numbers for independent tests of the loci, the second line is the combined regression for both loci.

\begin{tabular}{|c|c|c|c|c|c|c|c|c|c|c|}
\hline \multirow[b]{3}{*}{ Accession } & \multicolumn{10}{|c|}{ Locus } \\
\hline & \multicolumn{5}{|c|}{$B o C A L-a$} & \multicolumn{5}{|c|}{ BoAP1-a } \\
\hline & $n$ & $\beta$ & SD & $P(\beta \neq 0)$ & $r^{2}$ & $n$ & $\beta$ & $\mathrm{SD}$ & $P(\beta \neq 0)$ & $r^{2}$ \\
\hline PI 441510 & 35 & -0.69 & 0.27 & 0.016 & 0.168 & 34 & na $^{z}$ & & & \\
\hline PI 115881 & 39 & +0.13 & 0.27 & 0.62 & 0.008 & 32 & +0.14 & 0.27 & 0.60 & 0.009 \\
\hline loci combined y & 32 & +0.21 & 0.30 & 0.50 & & 32 & +0.23 & 0.30 & 0.45 & 0.024 \\
\hline PI 30928 & 38 & -0.25 & 0.17 & 0.15 & 0.056 & 38 & -0.48 & 0.32 & 0.14 & 0.060 \\
\hline loci combined $y$ & 38 & -0.23 & 0.17 & 0.18 & & 38 & -0.44 & 0.32 & 0.172 & 0.108 \\
\hline
\end{tabular}

¿Not applicable, this accession was monomorphic at BoAPl- $a$ for the mutant allele.

yFor combined analyses, partial coefficients are under each locus; the combined $R^{2}$ is under BoAPl-a. 


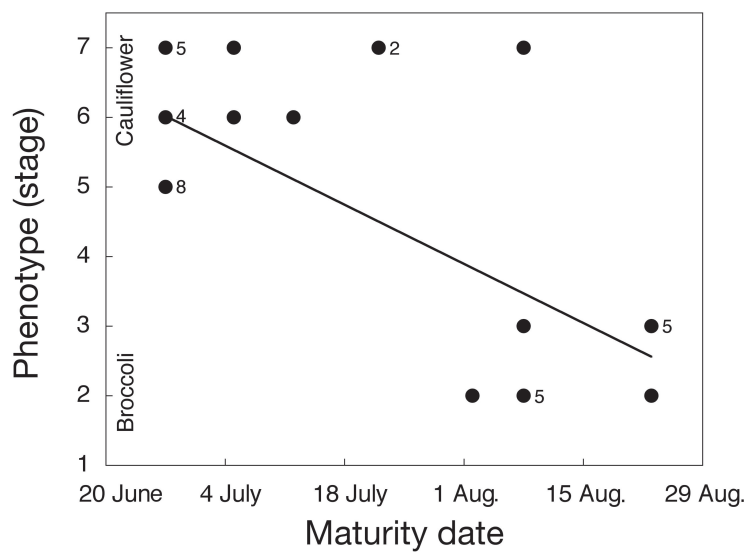

Fig. 3. Change in phenotype of accession G 30769 Brassica oleracea 'Green Harmony F1' over the course of the growing season when grown in the field at Geneva, N.Y. The phenotype was scored using the scale in Fig. 1. Numbers adjacent to data points indicate the number of observations. G 30769 is homozygous recessive for both $B o C A L-a$ and $B o A P 1-a$.

possibility that the phenotypic variation in 'Green Harmony F1' was a physiological response to temperature was tested by raising plants in the greenhouse and moving them to growth chambers at different temperatures at the time of reproductive transition. The association between temperature and arrest was reproduced (Fig. 4). Therefore, sensitivity to the environment can sometimes account for phenotypic variation within and among accessions. G 32210, 'High Sierra F1' behaved as a heat-injured broccoli. All G 32210 plants were homozygous mutant at both $B o C A L-a$ and $B o A P 1-a$, confirming the first year results that commercial broccoli can be monomorphic for mutations in both genes (Table 1). Broccoli hybrids that were homozygous mutant for $B o C A L-a$ can be phenotypically indistinguishable from standard broccoli (Fig. 5). The majority of plants in three accessions were too late in heading to collect sufficient phenotypic data for within-accession statistical tests in 2002; these were PI 462209 ('Broccolo Neri e Cespuglio'), PI 462210 ('Broccolo Natale Lopa'), and PI 462222 ('Violetto').

BoGSL-ELONG SEQUENCE. Nucleotide sequences of five homozygous wild type, five heterozygous, and five homozygous mutant BoGSL-ELONG genotypes confirmed visual scoring by length variation on agarose gels. This was done for quality control, to confirm that we were scoring the same gene as reported by $\mathrm{Li}$ and Quiros (2002). The observed length variation consisted of a 38-bp tandem repeat, present as one copy in the mutant and two copies in the wild-type allele. A representative sequence of each allele has been deposited in GenBank for broccoli accessions $G$ 30009 (homozygous mutant) and G 30415 (homozygous wild type) under accession nos. DQ445731 and DQ445730, respectively.

\section{Discussion}

For germplasm classification of broccoli and cauliflower, a genetic marker or a combination of markers that accurately predict phenotype would be valuable. Such markers are likely to be associated with genes that influence inflorescence development. We tested the association between genotype and phenotype for two genes believed to influence heading, BoAP1- $a$ and $B o C A L-a$ (Smith and King, 2000), as well as a gene involved in glucosinolate biosynthesis, BoGSL-ELONG (Li and Quiros, 2002). BoAP1-a and $B O C A L-a$ are transcription factors that function redundantly (Lowman and Purugganan, 1999). A nonfunctional allele at BoGSL-ELONG has been associated with white cauliflower, which lacks 4C GSL (Li and Quiros, 2002). For a diverse set of $B$. oleracea accessions, we tested simple genetic models of the three loci individually and $B o A P 1-a$ and $B O C A L-a$ combined. The goodness-of-fit test rejected the model that genotype is associated with predicted phenotype in all instances. BoAPI- $a$ and $B o C A L-a$ alleles or loci were significantly correlated with phenotype but the fraction of variation explained was low, $4.4 \%$ to $6.3 \%$. Adding $B o G S L-E L O N G$ to the analysis improved predictive power. The best three-locus model explained $24.8 \%$ of observed phenotypic variation. Although including BoGSL-ELONG in the model gave a substantial improvement, these three markers did not serve as robust predictive tools in this set of germplasm.

The genetic basis of heading phenotype should be more apparent within a line than among lines because cultivars reproduce true-to-type. Furthermore, BoAPl- $a$ and $B o C A L-a$ may have a greater effect in certain genetic backgrounds, as they do in the $\mathrm{N}$ $\times$ B mapping population (Smith and King, 2000). Therefore, we tested whether $B o C A L-a$ and/or BoAPl- $a$ predicted phenotype within accessions showing phenotypic or genotypic variation. BoCAL- $a$ and BoAPl- $a$ alleles had no effect in four accessions (G 30769, G 31824, G 32210, and HRI 5295) because the accessions were monomorphic for genotype or phenotype. Among the three segregating accessions, in only one did the genotype have a significant effect on phenotype. Within PI 441510 'Ramoso', $16.8 \%$ or $18.9 \%$ of phenotypic variation within accession could be explained by the $B o C A L-a$ locus, assuming additivity of the mutant allele or dominance, respectively $(P<0.01)$. Therefore, more of the phenotypic variation was explained by $B o C A L-a$ within 'Ramoso' than among the accessions compared in year 1 .

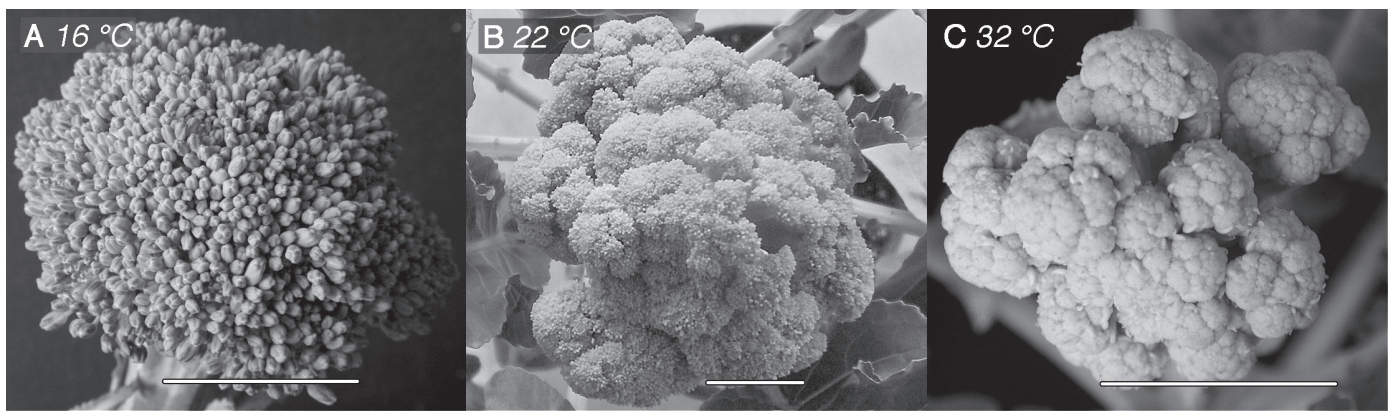

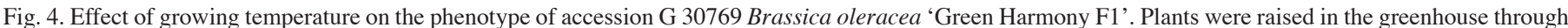

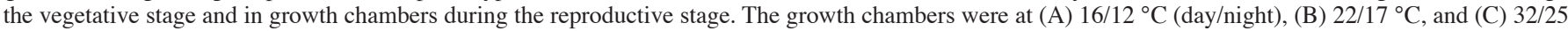
${ }^{\circ} \mathrm{C}$. Head size was limited by constraining rooting volume $(\mathrm{bar}=2 \mathrm{~cm})$. 


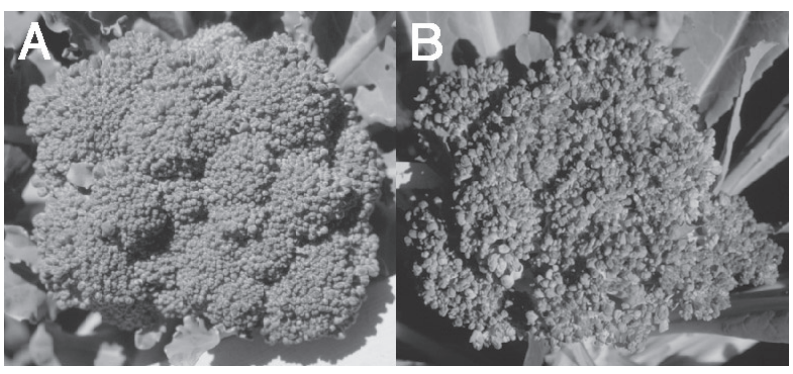

Fig. 5. Broccoli accessions homozygous for the mutant allele at the BoCAL- $a$ locus. (A) 'High Sierra', G 32210 (B) 'Ramoso', PI 441510. When grown in the field during the summer at Geneva, N.Y., these accessions showed no tendency to have an intermediate phenotype despite having the proposed cauliflower genotype.

If $B o A P 1-a$ and $B o C A L-a$ function redundantly in influencing phenotypic variation, their predictive value would be enhanced if they are analyzed in combination to account for epistasis. However, the combination of BoAPl-a and BoCAL- $a$ did not appreciably improve prediction. Additive models allowing for redundant gene function did not result in the genotype explaining a preponderance of the phenotypic variation. The model based on previous studies is that dominant wild-type alleles at $B o A P 1-a$ and $B o C A L-a$ produce a broccoli phenotype and recessive mutant alleles a cauliflower phenotype. That model is based both on gene function, the gene products are required for initiation of floral buds (Kempin et al., 1995; Lowman and Purugganan, 1999), and on genetics in model populations (Smith and King, 2000). BoGSL-ELONG has a mutant allele that had been observed solely in cauliflower ( $\mathrm{Li}$ and Quiros, 2002). A high frequency in cauliflower may be due to linkage drag, or coincidental selection, or there may have been selection based on product characteristics [e.g., attractiveness to insects (Raybould and Moyes, 2001)] associated with different amounts and types of glucosinolate. Many of the broccoli accessions in the present study carried the BoGSL-ELONG mutant allele at high frequency (Table 1).

In this paper, we followed the convention established by $\mathrm{Li}$ and Quiros (2002) in referring to the shorter BoGSL-ELONG allele as the mutant, because they associated it with improper splicing. However, our DNA sequence revealed that the length difference between the alleles is due to a tandem repeat. This result raises the possibility that the derived allele arose from a tandem duplication event rather than a deletion, and that what we refer to as the mutant allele is in fact ancestral.

Because tested genetic models did not hold robustly for this set of diverse accessions, it is likely that multiple genetic mechanisms can produce the broccoli phenotype. The effects of BoAPl- $a$ and $B o C A L-a$ could depend on genotypes at other loci or display high environmental sensitivity. The genetic model of broccoli and cauliflower derivation was developed using germplasm collected primarily in the center of origin for the crop (Smith and King, 2000). The collection in the present study consisted of materials produced by breeding programs worldwide. Associations in the ancestral populations have had ample opportunity to be lost when crosses were made and rigorous selection applied.

The utility of collections categorized by morphological traits, such as broccoli within $B$. oleracea, depends on accurate classification into categories. End-users of the collection may not be able to verify the trait. For instance, winter broccolis do not flower during the growing season in temperate climates, so users must rely on accurate passport data. Furthermore, curators benefit from efficient means of verifying the classification of newly acquired accessions or of accessions with questionable passport data.

High environmental sensitivity can result in misclassification in collections. 'Green Harmony F1' is a commercial hybrid cauliflower for the south Asian market, but it produces a typical broccoli head when grown for fall harvest in New York. It is possible that a double mutant genotype at BoAPl- $a$ and $B o C A L$ $a$ weakens the developmental signal for flower differentiation, and therefore makes such plants responsive to temperature cues whose effect is masked in wild-type genotypes.

Wild-type alleles at the BoAP1- $a$ and BoCAL- $a$ loci were associated with a cauliflower phenotype in some genetic backgrounds (Smith and King, 2000). In addition, Purugganan et al. (2000) observed the BoCAL- $a$ mutant allele in broccoli, kale, and wild cabbage. Our results showed that commercial broccoli accessions exist where bud formation is normal despite homozygosity of the mutant alleles at BoAPl- $a$ and BoCAL- $a$ loci. Therefore, other loci must be able to complement the roles of these genes in overcoming the arrested development normally associated with the $B o A P I-a$ and $B o C A L-a$ double mutant genotype and to determine whether a cultivar is broccoli or cauliflower. Our results overall indicate that in commercial cultivars, those complementary loci are more important in determining the horticultural type than are $B o A P 1-a$ and BoCAL- $a$.

\section{Literature Cited}

Colosi, J.C. and B.A. Schaal. 1993. Tissue grinding with ball bearing and vortex mixer for DNA extraction. Nucleic Acids Res. 21:10511052.

Crisp, P. 1982. The use of an evolutionary scheme for cauliflowers in the screening of genetic resources. Euphytica 31:725-734.

Crisp, P., D.G.A. Walkey, E. Bellman, and E. Roberts. 1975. A mutation affecting curd colour in cauliflower (Brassica oleracea L. var. botrytis DC). Euphytica 24:173-176.

Gray, A.R. 1982. Taxonomy and evolution of broccoli (Brassica oleracea var. italica). Economic Bot. 36:397-410.

Kalia, P. and S.R. Sharma. 2004. Current researches in hybrid broccoli, p. 109-134. In: P.K. Singh, S.K. Dasgupta, and S.K. Tripathi (eds.). Hybrid vegetable development. Food Products Press, Haworth Press, Binghamton, N.Y.

Kempin, S.A., B. Savidge, and M.F. Yanofsky. 1995. Molecular basis of the cauliflower phenotype in Arabidopsis. Science 267:522-525.

Li, G. and C.F. Quiros. 2002. Genetic analysis, expression and molecular characterization of BoGSL-ELONG, a major gene involved in the aliphatic glucosinolate pathway of Brassica species. Genetics 162:1937-1943.

Lowman, A.C. and M.D. Purugganan. 1999. Duplication of the Brassica oleracea APETELA1 floral homeotic gene and the evolution of domesticated cauliflower. J. Hered. 90:514-520.

Purugganan, M.D., A.L. Boyles, and J.I. Suddith. 2000. Variation and selection at the CAULIFLOWER floral homeotic gene accompanying the evolution of domesticated Brassica oleracea. Genetics 155:855-862.

Raybould, A. and C. Moyes. 2001. The ecological genetics of aliphatic glucosinolates. Heredity 87:383-391.

Smith, L.B. and G.J. King. 2000. The distribution of BoCAL-a alleles in Brassica oleracea is consistent with a genetic model for curd development and domestication of the cauliflower. Mol. Breeding 6:603-613.

Sokal, R.R. and F.J. Rohlf. 1981. Biometry: The principles and practice of statistics in biological research. 2nd ed. Freeman, New York. 